\title{
The effects of delay interval, intertrial interval, amnestic drugs, and differential outcomes on matching-to-position in rats
}

\author{
LISA M. SAVAGE and JOHN PARSONS \\ State University of New York, Binghamton, New York
}

\begin{abstract}
A matching-to-position task was used to investigate the effects of numerous variables on spatial working memory in the rat. The primary interest was whether implementing the differential outcomes procedure (DOP), which correlates specific reinforcers with specific discriminative stimuli, would enhance memory performance under a variety of conditions (long delay intervals, short intertrial-intervals [ITIs], amnestic drugs). When working memory was taxed, either by excessive delay intervals or very short ITIs, the DOP did enhance performance. Scopolamine disrupted performance in a delay-dependent manner, regardless of either reinforcement or ITI condition; however, performance was worse in the group that was trained with nondifferential reinforcement. MK801 also impaired performance, but the effect was different from that obtained with scopolamine. First, the effect of MK-801 was delay independent; second, the greatest disruption of performance was in the group of rats trained with differential reinforcement. These data suggest that scopolamine and MK-801 impair memory performance in very different ways, depending on the nature of the task.
\end{abstract}

A common procedure used to assess spatial working memory in rats is the operant version of matching-toposition (MTP; Blokland \& Dunnett, 1995; Bushnell, 1990; Dunnett, 1985, 1993; Dunnett \& Martel, 1990; Robinson \& Crawley, 1993). The differential outcomes procedure (DOP) has been shown to enhance performance on MTP tasks (Savage \& Langlais, 1995), as well as other conditional discriminations (Alling, Nickel, \& Poling, 1991a, 1991b; Demarse \& Urcuioli, 1994; Linwick, Overmier, Peterson, \& Mertens, 1988; Peterson \& Trapold, 1980; Savage, Stanchfield, \& Overmier, 1994; Trapold, 1970; Williams, Butler, \& Overmier, 1990). In the standard laboratory procedure, all correct choices in the matching-to-sample (MTS) or MTP task are reinforced with the same reinforcer - typically food. The DOP involves correlating each sample stimulus/correct choice combination with a distinct reinforcement condition. For example, one sample/choice combination may always lead to the delivery of food at the end of the trial, whereas the alternative combination might result in a different outcome such as the presentation of a $20 \%$ sucrose solution. This procedure allows for the learning of a predictive relation as to which reinforcer will be presented on the basis of the antecedent stimulus conditions. The common control procedure for such an experimental design is to use the same stimuli and reinforcers, but to randomize these relationships. Under these conditions, there is no predictive relation indicating which outcome will be given on the basis of the antecedent stimulus conditions.

Correspondence should be addressed to L. M. Savage, Department of Psychology, Binghamton University, P.O. Box 6000, Binghamton, NY 13902-6000.
The effect of using unique reinforcers correlated with each correct choice alternative, relative to either the traditional use of a common reinforcer for all correct choices or different but uncorrelated reinforcers, is that speed of learning the problem is increased and accuracy of memorybased performance across long delays is improved. This enhancement in learning and/or working memory is called the differential outcomes effect (DOE). It has been demonstrated in a variety of animal subjects such as rats, dogs, and pigeons (see Goeters, Blakely, \& Poling, 1992, for a review), as well as children (Maki, Overmier, Delos, \& Gutmann, 1995).

The DOP was first demonstrated to improve acquisition and memory performance in normal rats over two decades ago (Carlson \& Wielkiewicz, 1976; Fedorchak \& Bolles, 1986; Trapold, 1970), but its effect on the performance of brain-altered subjects has been tested only recently. Savage et al. (1994) found that the DOP used with symbolic and nonsymbolic MTS increased accuracy at long delays, even when pigeons were administered amnestic drugs. Savage and Langlais (1995) found that implementing the DOP improved acquisition on the T-maze version of MTP in rats with diencephalic lesions produced by pyrithiamine-induced thiamine deficiency.

In the present study, we implemented the DOP in the operant version of MTP to determine whether it would improve memory performance in normal rats under the following conditions: (1) an increase in the duration of temporal delay, (2) a decrease in intertrial interval (ITI), and (3) different amnestic drugs. It has been well established that increasing the length of delay interval and/or decreasing ITI results in decreased accuracy performance in rats trained on operant versions of MTP (Blok- 
land \& Dunnett, 1995; Dunnett, 1993; Dunnett \& Martel, 1990). However, there is still some question about whether various amnestic drugs produce delay-dependent or delay-independent effects on NMTP (non-matching-toposition) or MTP (Bushnell, 1990; Ennaceur, 1995). Delay-dependent behavioral deficits are more pronounced as delay interval increases - that is, under a drug condition, the performance will decline more rapidly as the delay interval increases. Delay-independent effects are those in which subjects are impaired at even the shortest delays (minimal working memory load) and in which accuracy performance under a drug state declines at a constant rate as delay interval increases. In this case, it has been hypothesized that the deficits are primarily due to non-mnemonic processes rather than working memory (Bushnell, 1990; Dunnett, 1985). However, this does not mean that a working memory impairment can be totally excluded (Ennaceur, 1995). The observed deficit may result from an interaction between impaired cognitive processes (e.g., increased distractability, visuospatial or perceptual deficits) and working memory.

The drugs chosen for this study-scopolamine, a cholinergic muscarinic antagonist, and MK-801, a glutamate noncompetitive antagonist at the NMDA receptorhave been shown to impair performance on some types of memory tasks. Previous research on working memory assessed by delayed MTP or delayed NMTP tasks without differential outcomes has shown that it is disrupted by both scopolamine (Dunnett, 1985; Hironaka, Hisatsugu, \& Kiyoshi, 1992; Kirkby, Jones, \& Higgins, 1995; Savage \& Overmier, 1995) and MK-801 (Cole, Klewer, Jones, \& Stephens, 1993; Pontecorvo, Clissold, White, \& Ferkany, 1991; Robinson \& Crawley, 1993). Some studies have reported that scopolamine and MK-801 produce similar (delay-independent) effects on memory tasksthus potentially sharing common mechanisms (Maurer, Storch, LaForge, \& Boast, 1995; Robinson \& Crawley, 1993). Other studies have demonstrated that MK-801 and scopolamine produce dissimilar impairments; specifically, scopolamine induces delay-dependent deficits, whereas MK-801 induces delay-independent deficits (Cohn, Ziriax, Cox, \& Cory-Slechta, 1992; Cole et al., 1993; Maurer et al., 1995; Ogura \& Aigner, 1993; Pontecorvo et al., 1991; Stanhope, McLenachan, \& Dourish, 1995). Numerous studies have reported that the effects of MK-801 are memory nonspecific and possibly due to increased perseveration (Cohn et al., 1992; Ogura \& Aigner, 1993; Stanhope et al., 1995). Other studies suggest the MK- 801 has more direct effects on memory processes, but the effects are task dependent (Caramanos \& Shapiro, 1994; Maurer et al., 1995; Mondadori \& Weiskrantz, 1993; Shapiro \& O'Connor, 1992). The differences in experimental findings, as well as interpretations, across studies are likely related to a number of task parameters (e.g., length of delay interval, location of nosepoke response manipulandum, dose of drug, method of drug delivery).

The use of a common task and the manipulation of a specific parameter within that task, such as differential outcomes versus nondifferential outcomes, to investigate the effects of lesions or drugs offers a unique opportunity for assessing dissociations. Any differences observed between groups in such a situation are likely to be a result of the specific parameter manipulation, rather than more global task differences. Thus, this type of procedural manipulation can be a powerful technique because the issue of task complexity or dramatic differences in task requirements, which is normally considered a problem with dissociation research (Olton, 1991; Roediger, 1991; Weiskrantz, 1968), cannot be implicated as a factor that secondarily influences the performance dissociations. The goal of the present study was to determine what type of dissociations would be produced by various parameter manipulations - including administration of amnestic drugs.

\section{METHOD}

\section{Subjects}

Sixteen naive adult male Sprague-Dawley rats, approximately 3 months old and $254 \mathrm{~g}$ on average at the start of the experiment, were used in the study. They were housed 2 to a cage with unlimited access to water and Purina rodent chow in a colony room with a 12:12-h light:dark cycle (onset at 7:00 a.m.). Two weeks prior to the onset of the experiment, the subjects' body weights were gradually reduced to approximately $80 \%$ of their free-feeding weights by restricted feedings. Their weights were maintained at this level by supplemental feedings of rodent chow (approximately $15 \mathrm{~g}$ ) in their home cages at the conclusion of each experimental session. The rats were allowed to gain weight $(5-10 \mathrm{~g}$ per week) relative to a normal growth curve (obtained from Harlan Sprague Dawley, Inc.).

\section{Apparatus}

The training and testing sessions were conducted in commercial small animal operant conditioning chambers $(29 \times 24 \times$ $30 \mathrm{~cm}$ ), each enclosed within a sound-attenuating chest (Med Associates Inc., St. Albans, VT). The front and back of the chamber were made of Plexiglas, whereas the ceiling and side walls were constructed of aluminum. An aperture $(5 \times 5 \mathrm{~cm})$ for accessing the food/water dispenser was centrally located on the front wall $1.6 \mathrm{~cm}$ above the grid floor. A photobeam sensor was inserted in the food/water aperture to detect head entry. The two response levers $(5 \mathrm{~cm}$ wide) were located $3 \mathrm{~cm}$ to either side of the food hopper and $6.3 \mathrm{~cm}$ from the grid floor. A panel light was located $6.3 \mathrm{~cm}$ above the center of the aperture containing the food/water dispensers. General illumination was provided by a houselight located in the middle of the back wall opposite the food/water dispenser, $24 \mathrm{~cm}$ above the grid floor. Background white noise $(80 \mathrm{~dB})$ was presented throughout the experimental sessions by a speaker located on the back wall. Experimental events were controlled and data were collected using a PC (Gateway 486) and Med PC interface and software (Med Associates, Inc., St. Albans, VT).

\section{Procedures}

\section{Pretraining}

Training was initiated with three sessions of habituation to the experimental chamber. During this phase, the subjects were placed in the chambers for $45 \mathrm{~min}$ with both the houselight and white noise activated. During Sessions 4 and 5, the rats were trained to eat and drink from the aperture. Each 45-min magazine training session included 48 trials with the random presentation (variableinterval [VI] $22.5 \mathrm{sec}$ ) of either a $45-\mathrm{mg}$ food pellet (BioServ, Frenchtown, NJ) or $0.1 \mathrm{cc}$ sucrose $(20 \%)$ water.

Next, the rats were trained to leverpress. Sixty seconds after the onset of the session, either the left or right lever (randomly deter- 
mined) was extended. Once the rats pressed the extended lever, either a food pellet or $0.1 \mathrm{cc}$ of sucrose water was presented (randomly determined). Each session included 48 trials with a VI 32.5-sec ITI. The rats were rewarded on a FR-1 schedule for two sessions. Animals that would not engage in leverpressing under that schedule were put on a standard autoshaping procedure for two sessions. During autoshaping, after the 60 -sec ITI, either the left or right lever (randomly determined) was extended for $8 \mathrm{sec}$ and then retracted. Immediately following the retraction of the lever, either a food pellet or sucrose solution (randomly determined) was presented. Both lever extension and food presentation were independent of the animal's behavior. Each session consisted of 48 trials. After autoshaping, the animals were transferred back to the FR-1 schedule. Once the rats had learned to leverpress on the FR-1 schedule, they were trained on an FR-2 schedule for two sessions. All animals were trained to leverpress on the FR-2 schedule within 8 days.

\section{Differential vs. Nondifferential Outcomes}

At the initiation of MTP training, and during all subsequent phases of the experiment, the DOP was employed. Prior to MTP training, animals were randomly assigned to either a differential $(n=8)$ or nondifferential $(n=8)$ reinforcement condition. Animals in the differential reinforcement (DIFF) group received one 45-mg food pellet as a reinforcer when the "sample" was the right lever and they chose the right lever during the choice phase; if the "sample" was the left lever, and they chose the left lever during the choice phase, they would receive $0.1 \mathrm{cc}$ of sucrose solution as the reinforcer. Animals in the nondifferential (ND) group received a random presentation of either reinforcer (pellet, sucrose solution) after the correct completion of a trial. These experimental groups were maintained throughout the remainder of the study.

\section{MTP Training}

MTP training comprised two phases, a sample phase and a choice response phase. During the sample phase, either the right or left lever was extended (semirandomly determined, for each lever would be selected for $50 \%$ of the trials), which the animal was required to press. The subject had $15 \mathrm{sec}$ to respond to the sample lever (limited hold); if a subject did not press the lever within $15 \mathrm{sec}$, the lever was retracted and a $30 \mathrm{-sec}$ ITI was initiated. After the ITI, the same sample lever was again inserted into the chamber. Surpassing the limited hold was not counted as an error trial. Once the lever was pressed in the sample phase (register of sample), it was retracted and the panel light was turned on. The panel light remained on until a nosepoke was made into the food/water aperture, at which time the panel light was turned off and both levers were extended for the choice response phase. The animal was required to press the lever that had been extended in the sample phase. The limited hold during this phase was the same as that in the sample phase, and a failure to respond was not counted as an error trial. The trial was recycled if the limited hold was surpassed. Once the rat made a choice, both levers were retracted. If a correct choice was made, the panel light was again turned on and remained on during the duration of the reinforcer delivery $(0.5 \mathrm{sec}$ for pellets, $8 \mathrm{sec}$ for sucrose water $)$. In the event of an incorrect choice, all lights, including the houselight, were turned off for $10 \mathrm{sec}$. All error trials were repeated until the animal made the correct choice. All trials with an error, including repeated trials, were scored as incorrect. This was done to control for side-biased responding. Thus if a subject was responding to only one lever, it would have an accuracy score of less than $50 \%$; if the animal responded on only one of the levers, it would have an accuracy score of $0 \%$. To evaluate the role of side bias, the number of error trials on each lever was recorded and analyzed. The ITI at this phase of training was VI-30 sec, and each session included 60 trials. MTP training was concluded when each animal achieved $90 \%$ accuracy for 3 consecutive days.

\section{Delayed Matching-to-Position}

(A) Standard procedure. Once the rats had reached criterion on the MTP procedure, the delayed MTP (DMTP) was begun. DMTP training was identical to the MTP procedure except that a delay of $0,2,4,8,16$, or $32 \mathrm{sec}$ (randomly selected) was introduced between the conclusion of the sample phase and the beginning of the choice response phase, and the number of trials per session was increased to 72 . If all trials were not completed in $90 \mathrm{~min}$, the session was terminated. After the presentation and response to the sample lever, the delay interval was initiated. During the delay interval, the panel light was turned on and, as during MTP training, the rat was required to make a nosepoke into the food hopper. The first nosepoke after the completion of the scheduled delay period terminated the delay period, at which time the panel light was turned off and both levers were extended into the chamber (choice phase). This procedure has been demonstrated to keep the rat continuously making nosepoke responses to the food/water aperture throughout the delay interval (Dunnett \& Martel, 1990). The time from the beginning of "set delay" $(0,2$, $4,8,16,32 \mathrm{sec}$ ) to the time the subject made a response during the choice phase was recorded as "true delay" (off set of the VI schedule and response on one of the choice levers). As in MTP training, a correct choice resulted in reinforcement dependent on group assignment (DIFF, ND), whereas an incorrect choice resulted in the panel light being turned off for $10 \mathrm{sec}$ followed by a repeat of the previous trial.

(B) Pharmacological testing with long (VI 30-sec) ITI. All rats were continued for 10 sessions on the DMTP with 72 trials per day and a VI 30-sec ITI. If all trials were not completed in $90 \mathrm{~min}$, the session was terminated. Only sessions with at least 60 trials were used in the analyses. Two drugs with amnestic effects scopolamine and MK-801-were used. All the injections were given intraperitoneally $30 \mathrm{~min}$ prior to the testing session and were in a volume of $1.0 \mathrm{ml} / \mathrm{kg}$ of body weight. Recovery days, on which no injections occurred but on which behavioral sessions were conducted, intervened between drug testing days until performance recovered.

Scopolamine. The muscarinic antagonist scopolamine hydrobromide (Sigma, St. Louis, MO) and its analogue, methyl scopolamine (Sigma), which does not as readily cross the blood-brain barrier, were dissolved in saline. Subjects were tested under (1) the saline vehicle, (2) $0.50 \mathrm{mg} / \mathrm{kg}$ of methyl scopolamine, and (3) 0.125 , 0.25 , and $0.50 \mathrm{mg} / \mathrm{kg}$ of scopolamine in a semirandom order.

$M K-801$. The competitive glutamate antagonist MK-801 (Research Biochemicals International, Natick, MA) was dissolved in saline. Subjects were tested under (1) the saline vehicle and (2) $0.05,0.125$, and $0.18 \mathrm{mg} / \mathrm{kg}$ of MK -801 in semirandom order.

(C) Variation of ITI procedure. Four sessions after the completion of pharmacological testing on the long ITI procedure, the effects of shortening the ITI on behavior were assessed. The following $A B A B$ procedure was conducted: (1) session of VI $30-\mathrm{sec}$ ITI, (2) session of VI 7-sec ITI, (3) session of VI 30-sec ITI, (4) session of VI 7-sec ITI. Each session, regardless of ITI, included 72 trials with the delay intervals of $0,2,4,8,16,32$, and $48 \mathrm{sec}$. The delay intervals were randomly distributed across trials within a session.

(D) Pharmacological testing with short (VI 7-sec) ITI. After the ABAB procedure varying ITI, all rats were continued for 10 sessions on the VI 7-sec ITI procedure. Using the same protocol as the long ITI procedure, with the exceptions of decreasing the ITI and adding an additional longer delay interval ( $48 \mathrm{sec})$, injections of scopolamine and MK-80I were given to all rats.

\section{RESULTS}

The present study employed a one between-subjects (differential reinforcement vs. nondifferential reinforcement), two within-subjects (condition [ITI or dose], 
A DIFFERENTIAL OUTCOMES

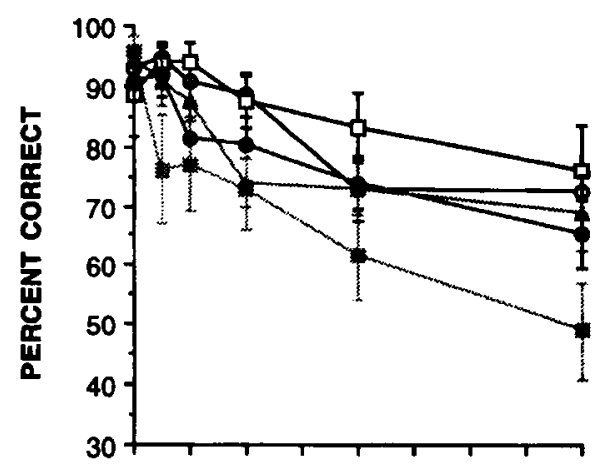

B NONDIFFERENTIAL OUTCOMES

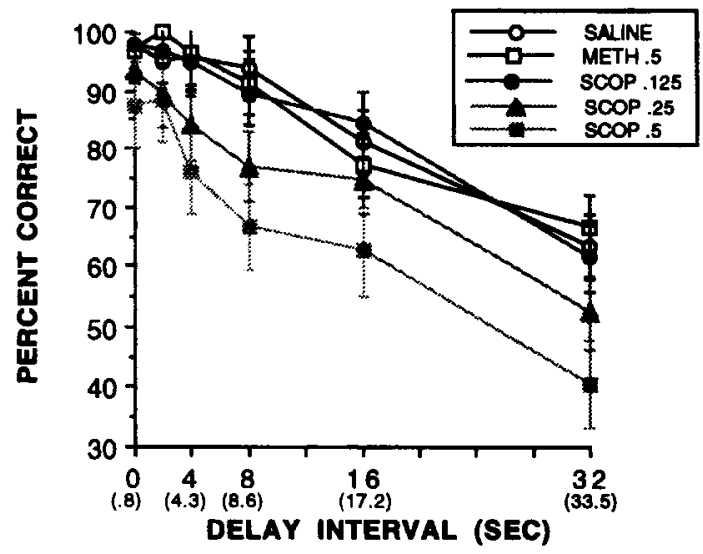

Figure 1. The effect of scopolamine and methyl scopolamine on memory choice accuracy on the delayed-matching-to-position task with a variable-interval 30 -sec intertrial interval (long ITI procedure). Panel A represents the data from the group trained with differential outcomes (DIFF group); panel B represents the data from the group trained with nondifferential outcomes (ND group). The numbers in parentheses on the $x$-axis (delay interval) represent the actual time between when the rats pressed a lever in the sample phase and when the rats pressed a lever in the choice phase. Error bars represent \pm 1 SEM.

delay interval) repeated measures analysis of variance (ANOVA) design. This statistical model was used to analyze both the accuracy data and the choice response time data (true delay interval data). The side-bias data were analyzed using a one between-subjects (differential reinforcement vs. nondifferential reinforcement) and one within-subjects (drug dose) repeated measures ANOVA. The data were analyzed using Super ANOVA software. A priori orthogonal planned tests between groups were conducted using $t$ tests (Howell, 1987; Kirk, 1982).

\section{Acquisition}

There were no significant group differences in the number of trials required to master the task $[F(1,14)=$ $0.016]$. Each group took approximately six sessions $[\mathrm{DIFF}=352$ (mean number of trials) \pm 17.7 (SEM);
$\mathrm{ND}=345 \pm 56.41]$ to reach the criterion of three sessions with above $90 \%$ correct choice accuracy.

\section{Pharmacological Testing During the Long ITI (30 VI) Procedure}

\section{Scopolamine}

There was no significant main effect of group $[F(1,14)=$ $0.37]$. In general, increases in both delay interval $[F(4,56)=$ $9.58, p<.0001]$ and drug dose $[F(5,70)=79.36, p<.0001]$ decreased performance. However, both of these factors were involved in interactions. Collapsed across all drug conditions, there were differences between the DIFF and ND groups as a function of delay interval [group $\times$ delay interaction: $F(5,70)=2.60, p<.05$ ]. Animals in both groups showed a significant decrease in performance as delay interval was increased. However, the ND animals showed slightly better performance $(<4.7 \%)$ than did DIFF animals at all delay intervals except $32 \mathrm{sec}$, where the relationship was reversed, and the DIFF group had higher accuracy scores than did the ND group (7.2\% higher). There was also a significant dose $\times$ delay interaction $[F(20,280)=1.95, p<.03]$. In both groups, there was very little difference in performance during the administration of saline, methyl sco-polamine, and $0.125 \mathrm{mg} / \mathrm{kg}$ of scopolamine. Performance in the DIFF group did not drop below $70 \%$ until subjects were administered the high dose of scopolamine. Then accuracy dropped to $60 \%$ at the 16 -sec delay interval and about $50 \%$ at the 32 -sec delay interval. In the ND group, performance after the administration of the medium dose of scopolamine dropped to $60 \%$ at the $32-\mathrm{sec}$ delay interval. Under the high dose of scopolamine, performance in the ND group dropped to about $62 \%$ at the 16 -sec delay interval and well below $50 \%$ at the 32 -sec delay interval.

Figure 1 shows that rats in both groups were proficient at responding to the set delay intervals. There were no group or dose differences (all $F \mathrm{~s}<1.3$ ) in the "true delay" time. There was a significant interaction for group $\times$ dose $\times$ lever $[F(4,56)=4.72, p<.01]$ on the side-bias error score. Although both groups tended to have an equal number of errors, the ND group equally distributed their mistakes across the left and right levers, whereas the subjects in the DIFF group made most of their errors when the correct response was the left lever.

Table 1

Side-Bias Data: Average Number of Errors That Occurred on Each Lever as a Function of Intertrial Interval Length

\begin{tabular}{lccccr}
\hline & \multicolumn{3}{c}{ ITI } & \multicolumn{2}{c}{ Short } \\
\cline { 2 - 3 } \cline { 5 - 6 } Lever & DIFF & ND & & DIFF & N D \\
\hline Right & 1.0 & 1.5 & & 0.8 & 4.9 \\
Left & 2.8 & 3.0 & & 6.6 & 7.6 \\
Total & 3.8 & 4.5 & & 7.4 & 12.5 \\
\hline
\end{tabular}

Note-DIFF, differential reinforcement; ND, nondifferential reinforcement. An error occurs when a subject chooses the wrong lever during the choice phase (i.e., if the sample was the left lever and a subject chose the right lever during the choice phase, it would be counted as a left lever error). 
Table 2

Side-Bias Data: Average Number of Errors That Occurred on Each Lever, as a Function of Intertrial Interval Length and Drug Dose

\begin{tabular}{|c|c|c|c|c|c|c|c|c|c|c|c|c|c|c|c|c|c|c|c|c|}
\hline \multirow[b]{4}{*}{ Lever } & \multirow{2}{*}{\multicolumn{10}{|c|}{ Long }} & & & & & & & & & & \\
\hline & & & & & & & & & & & \multicolumn{10}{|c|}{ Short } \\
\hline & \multicolumn{2}{|c|}{ Saline } & \multicolumn{2}{|c|}{ Meth } & \multicolumn{2}{|c|}{ Low } & \multicolumn{2}{|c|}{ Medium } & \multicolumn{2}{|c|}{ High } & \multicolumn{2}{|c|}{ Saline } & \multicolumn{2}{|c|}{ Meth } & \multicolumn{2}{|c|}{ Low } & \multicolumn{2}{|c|}{ Medium } & \multicolumn{2}{|c|}{ High } \\
\hline & DIFF & ND & DIFF & ND & DIFF & ND & DIFF & ND & DIFF & ND & DIFF & ND & DIFF & ND & DIFF & ND & DIFF & ND & DIFF & $\mathrm{ND}$ \\
\hline \multicolumn{21}{|c|}{ Scopolamine } \\
\hline Right & 1.5 & 4.6 & 3.9 & 3.3 & 1.0 & 5.6 & 1.3 & 7.8 & 2.6 & 13.8 & 1.0 & 5.1 & 1.0 & 3.9 & 1.8 & 5.8 & 0.9 & 7.6 & 3.4 & 10.5 \\
\hline Left & 9.8 & 4.0 & 5.4 & 5.5 & 9.6 & 3.4 & 10.9 & 6.9 & 17.8 & 7.5 & 3.9 & 5.4 & 4.9 & 9.0 & 5.8 & 6.4 & 7.4 & 7.4 & 15.6 & 10.6 \\
\hline Total & 11.3 & 10.6 & 9.3 & 8.8 & 10.6 & 9.0 & 12.2 & 14.7 & 20.4 & 21.3 & 4.9 & 10.5 & 5.9 & 12.9 & 7.6 & 12.2 & 8.3 & 15.0 & 19.0 & 21.1 \\
\hline \multicolumn{21}{|c|}{ MK-801 } \\
\hline Right & 0.3 & 2.5 & & & 1.0 & 1.5 & 1.3 & 3.3 & 2.6 & 6.6 & 1.3 & 5.9 & & & 0.5 & 5.4 & 0.5 & 5.0 & 0.0 & 6.4 \\
\hline Left & 4.6 & 2.5 & & & 3.6 & 1.9 & 15.6 & 3.5 & 10.3 & 7.6 & 3.5 & 5.9 & & & 6.6 & 4.0 & 11.3 & 3.4 & 16.9 & 4.5 \\
\hline Total & 4.9 & 5.0 & & & 4.6 & 3.4 & 16.9 & 6.8 & 20.9 & 14.2 & 4.8 & 11.8 & & & 7.1 & 9.4 & 11.8 & 8.4 & 16.9 & 10.9 \\
\hline
\end{tabular}

Note-DIFF, differential reinforcement; ND, nondifferential reinforcement. An error occurs when a subject chooses the wrong lever during the choice phase (i.e., if the sample was the left lever and a subject chose the right lever during the choice phase, it would be counted as a left lever error).

This bias was exaggerated as the dose of scopolamine was increased. (See Table 2.)

\section{MK-801}

Although there was no main effect of group $[F(1,14)=$ 2.67], there were significant main effects of dose $[F(3,42)$ $=11.33, p<.0001]$, and delay interval $[F(5,70)=12.49$, $p<.0001]$. There were no significant interactions. There was no effect of the low dose of MK-801 in either group. In the DIFF group, the medium and high doses of MK801 produced decrements in performance relative to saline, which was used as the reference condition [both $t \mathrm{~s}(42)>4.18, p<.01]$. Only the high dose of MK-801 caused a decrement in performance in the ND group, $[t(42)=2.47, p<.05]$. Figure 2 demonstrates that in both groups, the effect of a high dose of MK-801 was prevalent at all delay intervals. The significant effect of delay can be attributed to the data obtained under the saline condition; performance at the 32-sec delay interval was decreased relative to performance at the short delay intervals [all $t \mathrm{~s}(45)>2.31, p<.05$ ].

There were no group differences in true delay values. Likewise, there was no significant main effect for group on the side-bias error score (Table 2), but there were significant main effects for dose $[F(3,42)=6.64, p<.01]$ and lever $[F(1,14)=10.27, p<.01]$. The high dose of MK-801 $(0.18 \mathrm{mg} / \mathrm{kg})$ resulted in an overall increased collapsed delay value ( $10.58 \mathrm{vs} .11 .95 \mathrm{sec})$. The group $\times$ lever interaction approached significance $[F(1,14)=$ $4.37, p<.055]$. Overall errors increased as dose increased; however, the ND group did not have as great a disparity between errors on the left and right levers ( $3.72 \mathrm{vs.} \mathrm{5.88)}$ as the DIFF group (0.53 vs. 10.78 ).

\section{ITI (30 VI vs. 7 VI) Change}

The switch from long ITI to short ITI in the ABAB design produced main effects of group $[F(1,14)=8.58, p=$ $.01]$, ITI length $[F(1,14)=10.58, p<.01]$, and delay
$[F(6,84)=80.78, p<.0001]$; combined, however, these variables created a significant three-way interaction $[F(6,84)=2.3, p<.05]$. Regardless of the ITI length, there were no significant group differences at the delay interval of $16 \mathrm{sec}$ or lower [all $t \mathrm{~s}(84)<1.56$ ], but the DIFF group had superior performance relative to the ND group at the 32- and 48-sec delay intervals [both $t \mathrm{~s}(84)>$ $2.09]$. In terms of performance within groups, varying the ITI had no effect at any delay interval in the DIFF group [all $t \mathrm{~s}(84)<1.88$ ]. This was not the case for subjects in the ND group. At the short delay intervals, there were no significant differences [all $t \mathrm{~s}(84)<1.11$ ] between the short or long ITI sessions observed in the ND group. However, when transferred to the short ITI sessions, performance of the ND group fell significantly at the 32- and 48 -sec intervals [both $t \mathrm{~s}(84)>4.13, p<.025$ ]. Thus, the three-way interaction was due to the fact that performance of the DIFF group remained unchanged, at any delay interval, as a function of reducing the ITI, whereas the performance of the ND group was not affected by reducing the ITI until the delay interval was $32 \mathrm{sec}$ or more. This demonstrated that the DOP diminishes the proactive interference caused by reducing the ITI (Figure 3 ).

There was a significant group $\times$ ITI $\times$ delay interaction on the true delay time $[F(1,14)=5.64, p<.05]$. When switched to the short ITI, the DIFF group had slightly longer true delay values at the $8-(9.18$ vs. 8.27$), 16-$ (19.17 vs. 16.57), and $32-(34.15$ vs. 33.2 ) sec delay intervals. However, since the DIFF group had higher accuracy at these intervals, the difference is not likely important.

Table 1 shows that side bias was prominent in both groups in the analysis of "where" (left or right lever) the errors occurred. Both groups made fewer error responses on the right lever (2.03) relative to the left lever (5.0), and this was reflected in the significant main effect of lever $[F(1,14)=16.47, p<.001]$. The side bias toward more errors on the left lever was exaggerated when the ITI was decreased, particularly in the ND group [Group $\times$ ITI: $F(1,14)=5.36, p<.05]$. 
A DIFFERENTIAL OUTCOMES

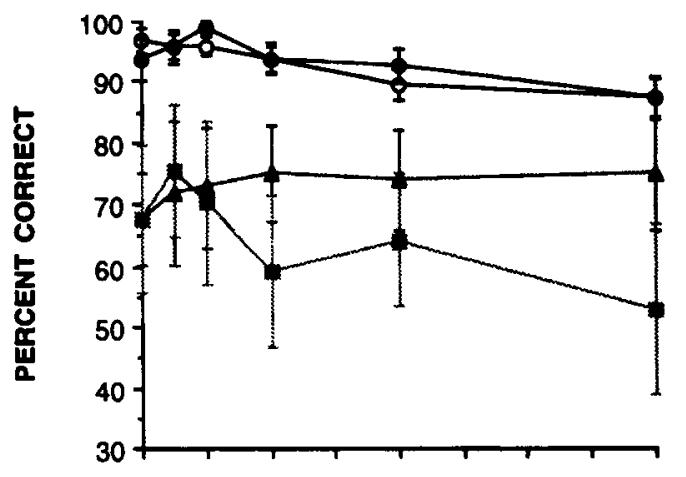

B NONDIFFERENTIAL OUTCOMES

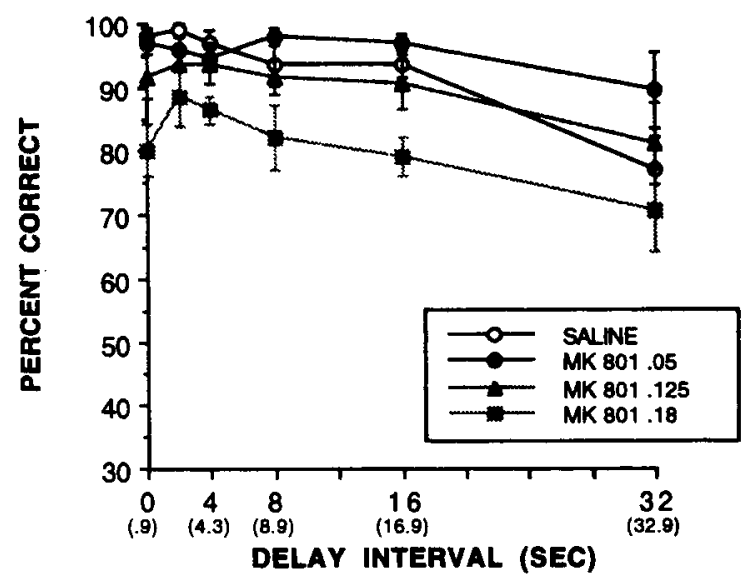

Figure 2. The effect of MK-801 on memory choice accuracy on the delayed-matching-to-position task with a variable-interval 30-sec intertrial interval (long ITI procedure). Panel A represents the data from the group trained with differential outcomes (DIFF group); panel B represents the data from the group trained with nondifferential outcomes (ND group). The numbers in parentheses on the $x$-axis (delay interval) represent the actual time between when the rats pressed a lever in the sample phase and when the rats pressed a lever in the choice phase. Error bars represent $\pm 1 S E M$.

\section{Pharmacological Testing During the Short ITI (VI 7) Procedure}

\section{Scopolamine}

There were significant main effects of group $[F(1,14)=$ $24.21, p<.001]$ and delay $[F(6,84)=64.66, p<.0001]$; however, the group $\times$ delay interaction was also significant $[F(6,84)=10.49, p<.0001]$. Both the DIFF and ND groups were able to perform with high levels of accuracy (above 90\%) at the short delays (Figure 4). However, as the delay interval increased to 32 and $48 \mathrm{sec}$, the performance of the ND group dropped to $50 \%$ or below, whereas the DIFF group continued to perform with a high level of accuracy [both $t \mathrm{~s}(84)<2.39, p<.05$ ]. This trend in performance was seen when subjects were administered saline, methyl scopolamine, and 0.125 and $.25 \mathrm{mg} / \mathrm{kg}$ doses of scopolamine. However, at the high- est dose of scopolamine $(0.5 \mathrm{mg} / \mathrm{kg})$, the performance of the DIFF group dropped off at the 8-sec delay interval and fluctuated between $60 \%$ and $70 \%$ at the longer delay intervals. In the ND group, at the highest dose of scopolamine, performance dropped to $60 \%$ at the $16-\mathrm{sec}$ delay interval and fell to chance levels at the 32- and 48sec intervals. This trend is reflected in the significant dose $\times$ delay interaction $[F(24,336)=2.77, p<.0001]$. Both groups had substantial decrements in performance under the high dose of scopolamine at the longer (8-, 16-, $32-, 48-\mathrm{sec})$ delay intervals [all $t \mathrm{~s}(336)>2.44, p<.05$ ].

There were no group or dose effects on true delay time. Both groups were able to match their response times to the set delay time. On the side-bias score, there was a significant interaction for group $\times$ dose $\times$ lever $[F(4,56)=$ $4.77, p<.01]$. Overall, the ND group made more errors than did the DIFF group (7.16 vs. 4.65 ). The subjects in the ND group tended to equally distribute their errors across the left and right levers, whereas subjects in the DIFF group made most errors on the left lever. This bias was exaggerated as the dose of scopolamine was increased (Table 2), but this was mostly due to the fact that subjects in the DIFF group were making very few errors with the saline, methyl scopolamine, or $0.125 \mathrm{mg} / \mathrm{kg}$ of scopolamine.

\section{MK-801}

There were no significant main effects for group $[F(1,14)=0.16$ or dose $[F(3,42)=1.51]$; combined, however, the two variables produced a significant interaction

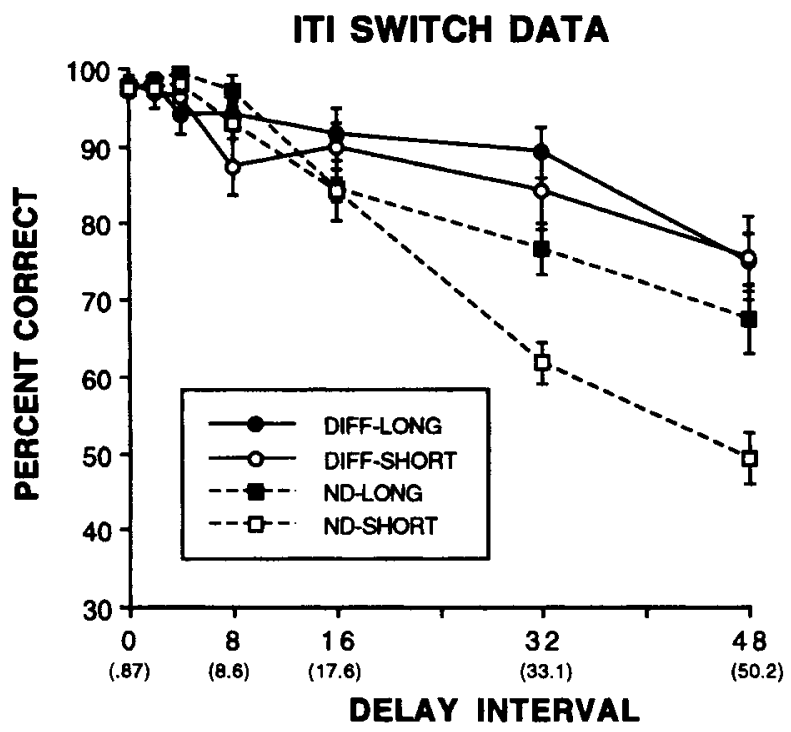

Figure 3. Choice accuracy as a function of the interaction between delay interval and intertrial interval (ITI) length. The circles represent the data from animals trained with the differential outcomes procedure (DIFF group), whereas the squares represent the data from the animals trained with the nondifferential outcomes procedure (ND group). Closed symbols represent data from the phase when the ITI was variable-interval (VI) 30 sec, and open symbols represent data from the phase when the ITI was a VI-7 sec. Error bars represent \pm 1 SEM. 
A DIFFERENTIAL OUTCOMES

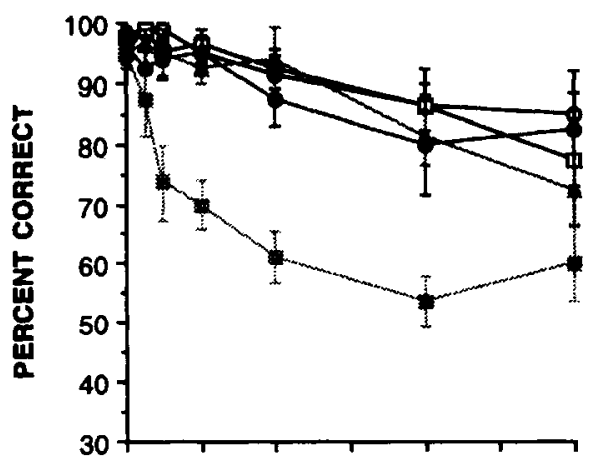

B NONDIFFERENTIAL OUTCOMES

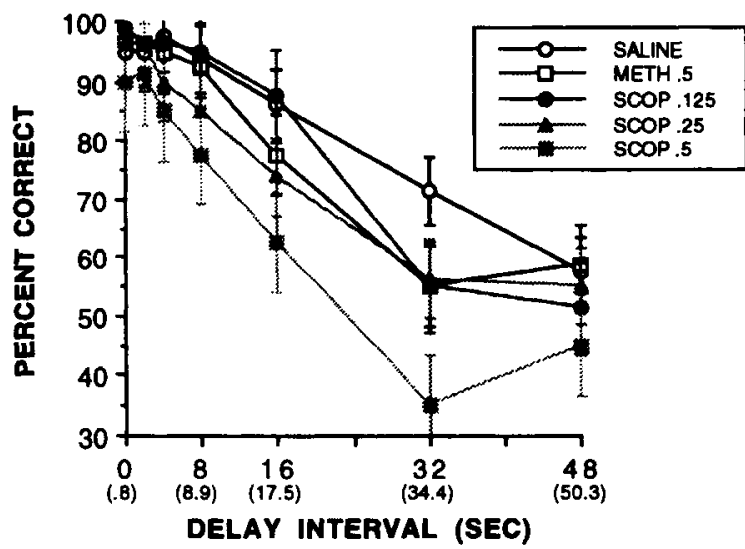

Figure 4. The effect of scopolamine and methyl scopolamine on choice accuracy on the delayed-matching-to-position task with a variable-interval 7-sec intertrial interval (short ITI procedure). Panel A represents the data from the group trained with differential outcomes (DIFF group); panel B represents the data from the group trained with nondifferential outcomes (ND group). The numbers in parentheses on the $x$-axis (delay interval) represent the actual time between when the rats pressed a lever in the sample phase and when the rats pressed a lever in the choice phase. Error bars represent $\pm 1 S E M$.

$[F(3,42)=3.53, p<.03]$. The high dose of MK-801 produced performance deficits among DIFF animals $[t(42)=$ $3.11, p<.05$ ] but not among ND animals. Again, the effect was delay independent, and performance at all delay intervals was impaired (Figure 5).

Accuracy in both groups generally declined with increasing delay intervals $[F(6,84)=45.48, p<.0001]$. However, increasing the delay interval had a much stronger detrimental effect on the ND animals than it did on the DIFF animals, resulting in a group $\times$ delay interaction $[F(6,84)=13.47, p<.001]$. In the saline and $0.05 \mathrm{mg} / \mathrm{kg}$ of MK-801 conditions, the ND group was more affected by the increasing delay interval.

Both groups were very good at adhering to the set delay values. The "true delay" values differed only slightly from the set delay intervals (Figure 5). There was a significant group $\times$ dose $\times$ lever interaction when the side-bias data were analyzed. Overall errors in- creased as dose increased in both groups, but the ND group did not have as many errors or as great a disparity between errors on the left and right levers as did the DIFF group (Table 2).

\section{DISCUSSION}

When a task is simple and subjects can easily solve it, there is no utilization of the DOP (Alling et al., 1991b; Savage \& Langlais, 1995). The present study supports these findings in that there was no benefit of using the DOP during acquisition or when short delay intervals were introduced between the sample and choice phases on the operant MTP task. However, when the delay interval was increased to sufficiently tax working memory and the ITI was reduced, the DOE was prominent.

\section{A DIFFERENTIAL OUTCOMES}
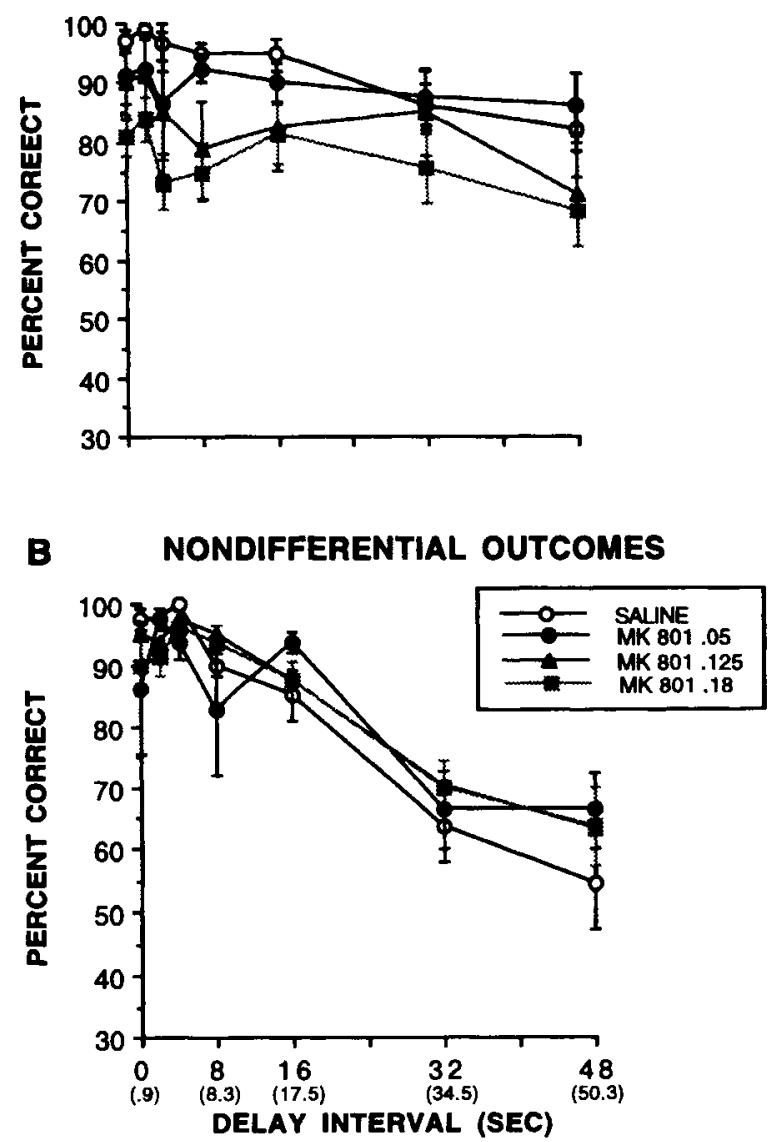

Figure 5. The effect of MK-801 on choice accuracy on the delayed-matching-to-position task with a variable-interval 7-sec intertrial interval (short ITI procedure). Panel A represents the data from the group trained with differential outcomes (DIFF group); panel B represents the data from the group trained with nondifferential outcomes (ND group). The numbers in parentheses on the $x$-axis (delay interval) represent the actual time between when the rats pressed a lever in the sample phase and when the rats pressed a lever in the choice phase. Error bars represent $\pm 1 S E M$. 
Reducing the ITI has been used as a procedure to increase proactive interference (Dunnett \& Martel, 1990; Dunnett, Martel, \& Iversen, 1990; Roitblat, 1980). Under a short ITI procedure animals are not as capable of "resetting" working memory, and information from the previous trial interferes with performance on the next trial (Roitblat, 1993). Short ITIs have been predicted to produce more errors because the animal is more likely to base its choice erroneously on its memory of the previous trial. Animals trained without the DOP are very sensitive to the decrease in ITI, whereas animals trained with the DOP are not affected at all. This suggests that the DOP not only strengthens the within-trial memory (see Trapold \& Overmier, 1972), but also allows for a better "resetting" of working memory.

The pharmacological challenge with scopolamine and MK-801 reveals two types of memory impairments. The first has to do with the nature of the impairments produced by the drugs regardless of reinforcement condition, and the second has to do with how reinforcement condition interacts with drug condition. As stated in the introduction, there are generally two types of impairments on tasks such as DMTS and DMTP: delay dependent and delay independent. There have been numerous studies over the years assessing the effects of scopolamine on performance on variations of the operant MTP task. Although all of these studies have reported a dose-dependent effect of scopolamine on impairing performance, there is some debate about the nature of the impairment. Some studies have reported a delay-dependent effect of scopolamine (Andrews, Jansen, Linders, \& Princen, 1994; Cole, Jones, \& Turner, 1994; Dunnett, 1985), whereas others have reported a delay-independent effect (Bushnell, 1990; Kirkby et al., 1995; Robinson \& Crawley, 1993).

In the present study, the effect of scopolamine was delay dependent. This was the case for both the DIFF group, upon which increasing delay interval in non-drug situations had relatively no effect, and in the ND group, for which increasing delay interval resulted in a dramatic decline in performance. The data in Figures 1 and 4 suggest that the rats trained with the DOP performed better than did rats trained with the ND procedure under nondrug conditions, as well as under the low and medium doses of scopolamine. Analysis of the error data across the doses of scopolamine revealed that more errors were made in the ND group than in the DIFF group. The pattern of errors across the two groups was different. Whereas subjects in the ND group distributed their errors across both response manipulanda, the subjects in the DIFF group made most, or all, of their errors on the left lever. This means that they tended to choose the lever associated with pellets (right) over that associated with sucrose solution (left). They tended to have this preference slightly even in a non-drugged state (Table 1). Thus, the scopolamine did not create the side-bias; rather, it exaggerated a preexisting preference.

The next challenge is to interpret the side-bias data. Were subjects in the DIFF group engaging in persevera- tive responding? If a subject is using a pure motoric strategy (i.e., perseveration) as a means of selecting correct choice alternatives, a response bias should occur at all delay intervals. This does not clearly occur in the scopolamine data; rather, subjects performed above $90 \%$ at the shortest delay. Thus the increase of perseverative responding is a function of delay interval-or memory capacity. This suggests that the DIFF animals were perseverating when they could not remember the correct response to initiate; thus they chose the response associated with the preferred reinforcer (see Savage et al., 1994, for data on reinforcer bias relative to side bias).

The MK-801 data turned out to be quite interesting. Like scopolamine, MK-801 has been used extensively to produce an "amnestic" effect on MTP tasks. Most studies have reported that MK-801 produces a delay-independent effect (Cole et al., 1993; Pontecorvo et al., 1991; Robinson \& Crawley, 1993; Stanhope et al., 1995). The data from both groups (DIFF and ND) in this study follow that delay-independent trend. Under the long ITI procedure, the $0.18-\mathrm{mg} / \mathrm{kg}$ dose of MK-801 impaired performance at all delay intervals in both groups. The DIFF group's performance also dropped at all delay intervals at the $0.125-\mathrm{mg} / \mathrm{kg}$ dose. In general, the effect of MK801 was more pronounced in the DIFF group. When retested and administered $0.18 \mathrm{mg} / \mathrm{kg}$ of MK- 801 under the short ITI procedure, there was virtually no effect upon choice performance in subjects trained with nondifferential reinforcement, but there was a disruption of delayed choice performance trained under differential reinforcement. This effect is opposite that of scopolamine, described earlier.

Analysis of the error data (Table 2) during the administration of MK-801 revealed that the ND group did not have a side bias, whereas the DIFF group did. In this case, unlike scopolamine, the side bias occurred in the DIFF group at all delay intervals, since the effect was delay independent. Thus it is likely that a perseverative response pattern contributed some to the deficit observed in the DIFF group. Again, however, it is difficult to determine whether the side bias causes the errors or whether the side bias is a result of poor memory. The subjects in the DIFF group were not using a perseveration strategy as their only behavioral response, or their percent correct score would have been well below 50\% (see Method section). Even though subjects tended to choose the right lever when they should have chosen the left, they were also making correct responses on the left lever. The perseverative or side-bias effects of MK-801 are task parameter specific, since we do not see this pattern of error in the ND group. No other motoric or other nonspecific factors influenced performance. Analysis of the true delay data is also a way of examining increases in response time. There was very little change in the "true delay" times between the groups or as drug dose was increased.

The finding that MK- 801 results in a dissociative effect complements the results obtained by other researchers, 
which have demonstrated that the effects of MK-801 are task dependent (Caramanos \& Shapiro, 1994; Maurer et al., 1995; Mondadori \& Weiskrantz, 1993; Shapiro \& O'Connor, 1992). Caramonos and Shapiro have suggested that "MK-801 produces an impairment in learning to represent environments" (p. 33). It has been known for some time (Peterson \& Trapold, 1980) that altering the DOP in well-trained animals by removing the correlation between sample and reinforcer (i.e., disruption of the stimulus-stimulus representation) causes performance to drop to chance levels. The decrements in performance seen after the administration of MK-801 in the DIFF group resemble data obtained from animals trained with the DOP and then exposed to the removal of the correlation between sample and reinforcer.

It has been suggested that the higher accuracy obtained under the differential outcomes procedures, relative to the nondifferential procedure, reflects either greater stimulus control (Alling et al., 1991a, 1991b) or a different memory mediation process (Linwick et al., 1988). If greater stimulus control is the mechanism by which DOP enhances performance, it would be expected that performance would be better in subjects trained with the DOP, regardless of the drug (Alling et al., 1991b; Thompson, 1978). Prior to the MK-801 data obtained in this study, previous research has supported that hypothesis. This is the first reported instance of a drug (MK-801) disrupting performance more in subjects trained with the DOP. Thus a simple stimulus control hypothesis cannot explain the data. However, if the DOP results in a different memory mediation process, there may be circumstances under which performance may be more disrupted by a drug or lesion in animals trained with the DOP.

Originally, when the DOE was discovered, Trapold and Overmier (1972) hypothesized that in the pairing phase, the subject learned something specific about the reward and that this "conditioned expectation" of the reward had stimulus-like or cue properties that guided specific acts. Such conditioned expectations are a kind of prospective memory-a memory elicited by the conditioned stimulus of what event comes next. Thus, subjects trained with the DOP can in principle solve these delay problems on the basis of either (1) their regular "retrospective" memory of what the discriminative cue was or (2) their elicited prospective memory - with its associated cue properties--of what the upcoming reward will be. The nondifferential or common outcome procedure affords only one source of information-the retrospective recall of what the discriminative stimulus sample was (see Linwick et al., 1988).

A two-memory process (prospective vs. retrospective) interpretation of the data would suggest that retrospective memory is substantially dependent on cholinergic mechanisms, but less so on glutamate-mediated mechanisms. On the other hand, prospective memory is more dependent on glutamate-mediated mechanisms than on cholinergic mechanisms.
In conclusion, the DOP does enhance memory performance under certain circumstances. Whether it can be implemented as a therapeutic aid to enhance memory performance appears to be dependent on the type of information that needs to be remembered (task parameters) and the nature of the memory disorder (type of neurochemical disruption).

\section{REFERENCES}

Alling, K., Nickel, M., \& Poling, A. (1991a). The effects of differential and nondifferential outcomes on response rates and accuracy under a delayed-matching-to-sample procedure. Psychological Record, 41, 537-549.

Alling, K., Nickel, M., \& Poling, A. (1991 b). The effects of phenobarbital on responding under delayed-matching-to-sample procedures with differential and nondifferential outcomes. Pharmacology, Biochemistry \& Behavior, 39, 817-820.

Andrews, J. S., Jansen, J. H., Linders, S., \& Princen, A. (1994). Effects of disrupting the cholinergic system on short-term spatial memory in rats. Psychopharmacology, 115, 485-494.

Blokland, A., \& DunnetT, S. B. (1995). Spontaneous response tendencies in noncontingent trials of matching-to-position tasks in rats: Consequences for learning the matching and nonmatching task contingencies. Psychobiology, 23, 76-84.

BushNELL, P. J. (1990). Modeling working and reference memory in rats: Effects of scopolamine on delayed matching to position. $B e$ havioral Pharmacology, 1, 419-427.

Caramanos, Z., \& Shapiro, M. L. (1994). Spatial memory and $N$ methyl-D-aspartate receptor antagonists APV and MK-801: Memory impairments depend on familiarity with the environment, drug dose, and training duration. Behavioral Neuroscience, 108, 30-43.

Carlson, J. G., \& WiElkiEwicz, R. M. (1976). Mediators of the effects of magnitude of reinforcement. Learning \& Motivation, 7 , 184-196.

Cohn, J., ZiriaX, J. M., CoX, C., \& Cory-Slechta, D. A. (1992). Comparison of error patterns produced by scopolamine and MK-801 on repeated acquisition and transition baselines. Psychopharmacology, 107, 243-254.

Cole, B. J., Jones, G. H., \& Turner, J. D. (1994). 5-HT1A receptor agonists improve the performance of normal and scopolamineimpaired rats in an operant delayed matching to position task. Psychopharmacology, 116, 135-142.

Cole, B. J., Klewer, M., Jones, G. H., \& Stephens, D. N. (1993). Contrasting effects of the competitive NMDA antagonist CPP and the non-competitive NMDA antagonist MK 801 on performance of an operant delayed matching to position task in rats. Psychopharmacology, 111, 465-471.

Demarse, T. B., \& URCuioli, P. J. (1994). Enhancement of matching acquisition by differential comparison-outcome associations. Journal of Experimental Psychology: Animal Behavioral Processes, 19, 317-326.

DunnetT, S. B. (1985). Comparative effects of cholinergic drugs and lesions of nucleus basalis or fimbra-fornix on delayed matching in rats. Psychopharmacology, 87, 357-363.

DUNNETT, S. B. (1993). Operant delayed matching and non-matching to position in rats. In A. Sahgal (Ed.), Behavioral neuroscience: $A$ practical approach (pp. 123-136). New York: Oxford University Press.

Dunnet T, S. B., \& Martel, F. L. (1990). Proactive interference effects on short-term memory in rats: Basic parameters and drug effects. Behavioral Neuroscience, 104, 655-665.

Dunnett, S. B., Martel, F. L., \& Iversen, S. D. (1990). Proactive interference effects on short-term memory in rats: II. Effects in young and aged rats. Behavioral Neuroscience, 104, 666-670.

ENNACEUR, A. (1995). The construct validity of animal models of memory: 1. Behavioral paradigms. International Behavioral Neuroscience Society Abstracts, 4, 30.

FEDorchaK, P. M., \& Bolles, R. C. (1986). Differential outcomes ef- 
fect using a biologically neutral outcome difference. Journal of Experimental Psychology: Animal Behavioral Processes, 2, 125-130.

GoETERS, S., Blakely, E., \& Poling, A. (1992). Differential outcomes effect. Psychological Record, 42, 389-412.

Hironaka, N., Hisatsugu, M., \& KiYoshi, A. (1992). Effects of psychoactive drugs on short-term memory in rats and rhesus monkeys. Japanese Journal of Pharmacology, 59, 113-120.

HowElL, D. C. (1987). Statistical methods for psychology (2nd ed.). Boston: Duxbury Press.

KIRK, R. E. (1982). Experimental design: Procedures for the behavioral sciences (2nd ed.). Belmont, CA: Wadsworth.

KIRKBY, D. L., Jones, D. N., \& HigGins, G. A. (1995). Influence of prefeeding and scopolamine upon performance in a delayed matchingto-position task. Behavioural Brain Research, 67, 221-227.

Linwick, D., Overmier, J. B., Peterson, G., \& Mertens, M. (1988) Interaction of memories and expectancies as mediators of choice behavior. American Journal of Psychology, 101, 313-334.

Maki, P., Overmier, J. B., Delos, S., \& GutmanN, A. J. (1995). Expectancies as factors influencing conditional discrimination performance of children. Psychological Record, 45, 45-71.

Maurer, S. A., StorCh, F. E., LaForge, R. R., \& BoAst, C. A. (1995) Task difficulty determines the differential memory-impairing effects of EAA antagonists in gerbils. Pharmacology, Biochemistry \& Behavior, 51, 345-351.

MONDADORI, C., \& WEISKRANTZ, L. (1993). NMDA receptor blockers facilitate and impair learning via different mechanisms. Behavioral \& Neural Biology, 60, 205-210.

Ogura, H., \& Aigner, T. G. (1993). MK-801 impairs recognition memory in rhesus monkeys: Comparison with cholinergic drugs. Journal of Pharmacology \& Experimental Therapeutics, 266, 60-64.

OltoN, D. S. (1991). Experimental strategies to identify the neurological bases of memory: Lesions. In J. L. Martinez \& R. P. Kesner (Eds.), Learning and memory: A biological view (pp. 441-463). New York: Academic Press.

Peterson, G., \& Trapold, M. (1980). Effects of altering outcome expectancies on pigeons' delayed conditional discrimination performance. Learning \& Motivation, 11, 267-288.

Pontecorvo, M. J., Clissold, D. B., White, M. F., \& Ferkany, J. W. (1991). $\mathrm{N}$-methyl-D-aspartate antagonists and working memory performance: Comparison with the effects of scopolamine, propranolol, diazepam, and phenylisopropyladenosine. Behavioral Neuroscience, 105, 521-535.

Robinson, J. K., \& CraWley, J. N. (1993). Intraventricular galanin impairs delayed non-matching-to-sample performance in rats. Behavioral Neuroscience, 107, 458-467.

ROEDIGER, H. L. (1991). Implicit memory: Retention without remembering. American Psychologist, 9, 1043-1056.
Rortblat, H. L. (1980). Codes and coding processes in pigeon shortterm memory. Animal Learning \& Behavior, 8, 341-351.

RoITBLAT, H. L. (1993). Representation and processes in working memory. In T. R. Zentall (Ed.), Animal cognition: A tribute to Donald A. Riley (pp. 175-192). Hillsdale, NJ: Erlbaum.

SavaGe, L. M., \& Langlais, P. J. (1995). Differential outcomes attenuate spatial memory impairments on matching-to-position following pyrithiamine-induced thiamine deficiency in rats. Psychobiology, 23, 153-160.

SAVAGE, L. M., \& OVERmier, J. B. (1995). The influence of sequential information in rats: Learning, memory and the effects of amnestic drugs. Learning \& Motivation, 26, 300-322.

Savage, L. M., STANChfield, M., \& Overmier, J. B. (1994). The effect of scopolamine, diazepam and lorazepam on working memory in pigeons: An analysis of reinforcement procedures and sample problem type. Pharmacology, Biochemistry \& Behavior, 48, 183-192.

ShaPIRo, M. L., \& O'CONNOR, C. (1992). $N$-methyl-D-aspartate receptor antagonist MK-801 and spatial memory representation: Working memory is impaired in an unfamiliar but not in a familiar environment. Behavioral Neuroscience, 106, 604-612.

Stanhope, K. J., McLenachan, A. P., \& Dourish, C. T. (1995). Dissociation between cognitive and motor/motivational deficits in the delayed matching to position test: Effects of scopolamine, 8-OHDPAT and EAA antagonists. Psychopharmacology, 122, 268-280.

THOMPSON, D. M. (1978). Stimulus control and drug effects. In D. E. Blackman \& D. J. Sanger (Eds.), Contemporary research in behavioral pharmacology (pp. 159-208). New York: Raven Press.

TRAPOLD, M. A. (1970). Are expectancies based upon different positive reinforcing events discriminably different? Learning \& Motivation, 1, 129-140.

Trapold, M. A., \& Overmier, J. B. (1972). The second learning process in instrumental learning. In A. H. Black \& W. F. Prokasy (Eds.), Classical conditioning II: Current research and theory (pp. 427-452). New York: Appleton Century Crofts.

WEISKRANTZ, L. (1968). Traps and pontifications. In L. Weiskranz (Ed.), Analysis of behavioral change (pp. 415-429). New York: Harper \& Row.

Williams, D. A., Butler, M. M., \& Overmier, J. B. (1990). Expectancies of reinforcer location and quality as cues for a conditional discrimination in pigeons. Journal of Experimental Psychology: Animal Behavioral Processes, 16, 3-13.

(Manuscript received August 15, 1996; revision accepted for publication June 3, 1997.) 